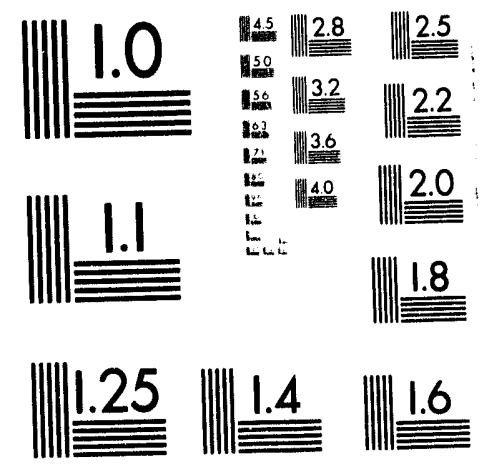



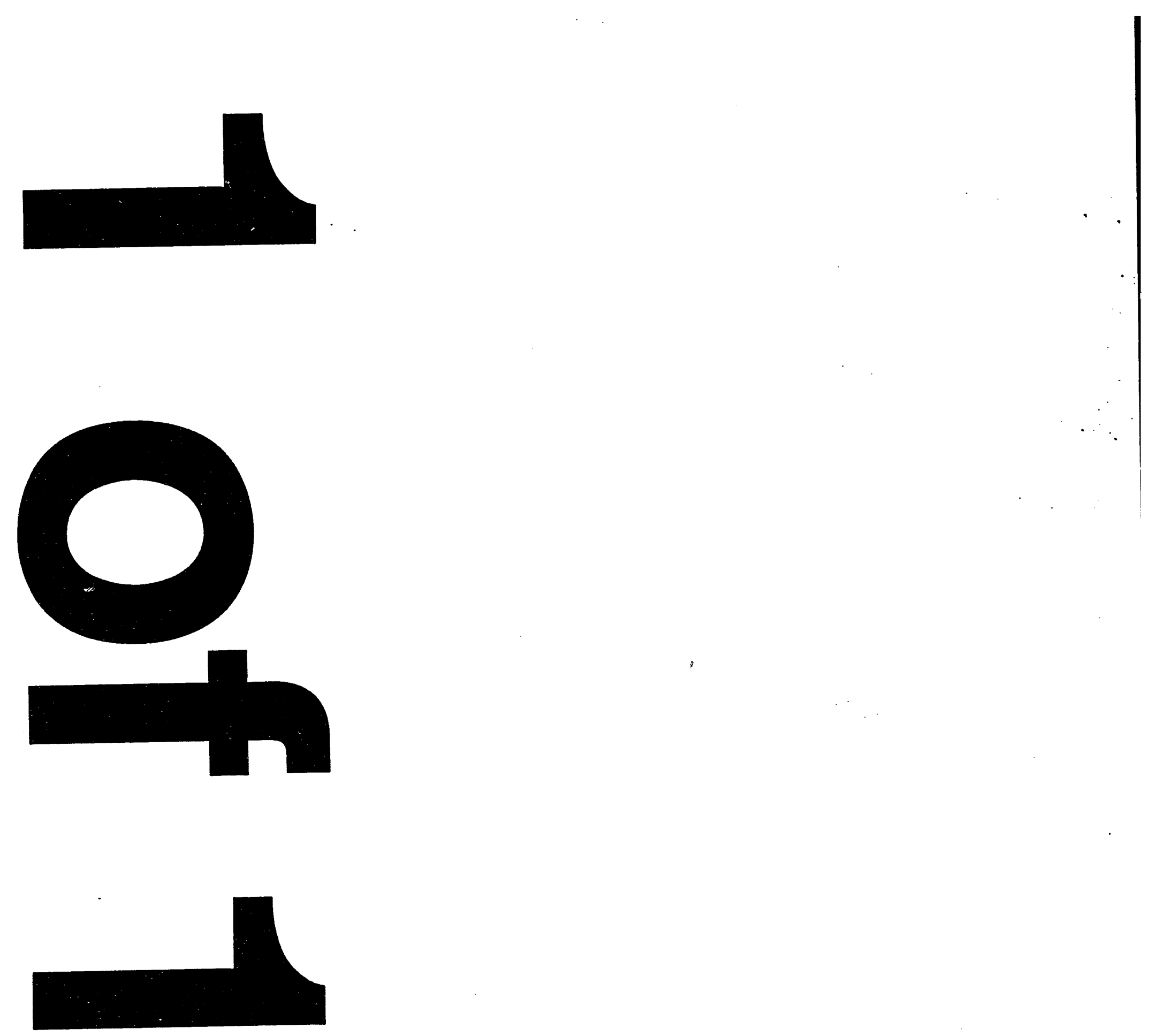
TITLE:

\section{SEQUENTIAL CLONING OF CHROMOSOMES}

Inventor:

Sanford A. Lacks

14 Highview Boulevard

Brookhaven, New York 11719

\section{Citizenship:}

\section{U.S.A.}

\section{DISCLAIMER}

This report was prepared as an account of work sponsored by an agency of the United States Government. Neither the United States Government nor any agency thereof, nor any of their employees, makes any warranty, express or implied, or assumes any legal liability or responsibility for the accuracy, completeness, or usefulness of any information, apparatus, product, or process disclosed, or represents that its use would not infringe privately owned rights. Reference herein to any specific commercial product, process, or service by trade name, trademark, manufacturer, or otherwise does not necessarily constitute or imply its endorsement, recommendation, or favoring by the United States Government or any agency thereof. The views and opinions of authors expressed herein do not necessarily state or reflect those of the United States Government or any agency thereof. 
The United States Government has rights in this invention pursuant to Contract No. DE-ACO2-76CH0016, between the United States Department of Energy and Associated Universities, inc.

\section{BACRGROUND OF THE INVENTION}

1. Field of Invention

This invention relates to a method for the sequential cloning of chromosomal DNA.

2. Background of Related Art

Currently, large sums of money are being spent and great efforts are being made to map the human genome and the genomes of other organisms. Such mapping is of great interest to molecular biologists.

Certain disorders, such as for example, hemophilia and Lou Gehrig's Disease, are associated with defective genetic material. One of the aims of the human genome mapping project is to discover which diseases are associated with defective genetic material. If the structure of such defective genetic material were known, reliable tests could be developed to determine, for example, who would be susceptible to certain forms of cancer, Lou Gehrig's Disease and other genetic disorders. 
The circular integration of plasmid DNA into a bacterial chromosome is described by Mejean et al. and by Niaudet et al.; see, Niaudet et al., "Insertional mutagenesis in Bacillus subtilis: mechanism and use in gene cloning", Gene, 19, 277-284 (1982); and Mejean et al., "Rapid cloning of specific DNA fragments of streptococcus pneumoniae by vector integration into the chromosome followed by endonucleolytic excision", Gene, 15, 289-293 (1981). Mejean et al. and Niaudet et al. furthrr disciose that when heterologous DNA in the circularly integrated plasmid have the ability to replicate in a different host bacterium, then a recombinant plasmid may be removed with restriction enzymes, ligated and cloned in that different host bacterium. However, the methods of Mejean et al. and Niaudet et al. are limited to the cloning of specific DNA fragments.

It is a purpose of the invention to provide a method for sequential cloning of genomic DNA.

Another purpose of the present invention is to provide a method for the sequential mapping of a genetic sequence.

still a further purpose of the present invention is to provide a method to be used in conjunction with the mapping of the human genome.

A method for sequentially synthesizing gene length segments of DNA is described in U.S. Patent No. 4,293,652. The method includes synthesizing a fragment of double 
stranded DNA corresponding to a preselected portion of the length of DNA to be synthesized or "cloned". A fragment of the preselected DNA is inserted into the cloning vector. The vector is cloned in an appropriate host. Next, the vector is opened by a restriction enzyme which cuts at the end of the first fragment. Another segment of the preselected DNA is then inserted into the vector. The process is repeated until the entire length of the preselected DNA has been inserted into the vector. The entire length of the preselected DNA may then be cloned by inserting the vector into a suitable host. This method sequentially synthesizes and clones an already known segment of DNA, unlike the method of the present invention, which sequentially clones and maps an unknown segment of DNA.

For a better understanding of the present invention reference is made to the following description, taken together with the accompanying drawings, and its scope will be pointed out in the appended claims.

\section{SUMMARY OF THE INVENTION}

The above mentioned and other purposes are achieved by the present invention, which includes a method for the sequential cloning of chromosomal DNA and, also, the chromosomal DNA cloned by this method. The method includes, as an initial step, selecting a target organism having a segment of chromosomal DNA to be sequentially cloned. Next, a short DNA segment homologous to a segment of the 
chromosomal DNA to be sequentially cloned is isolated. The homologous DNA segment has the first restriction site on either side of the segment. A first vector product is then formed by ligating the homologous segment into a DNA vector which cannot replicate within the target organism. The vector contains, in adjacent or overlapping positions, the first, second, and third restriction sites. The third restriction site is specific for a retrorestriction enzyme (classified as IIS).

The target organism is then transformed with the first vector product. During the transformation process, circular integration of the first vector product into the segment of DNA to be sequentially cloned occurs. The resulting recombinant chromosomal DNA segment includes two homologous DNA segments, one at either end of the inserted vector segment. The resulting recombinant chromosomal DNA is then cleaved with a restriction enzyme specific for the second restriction site to produce a cleaved DNA segment having the second restriction site at each end. The cleaved DNA segment, having the second restriction site at either end, is then circularized by ligation to form the second vector product.

Next, a host organism which is permissive for replication of the vector is transformed with the second vector product, so that the second vector product is selectively replicated. The replicated second vector 
product is cleaved with the first restriction enzyme and a DNA segment containing the vector and a short segment of DNA homologous to only a portion of the previously isolated DNA segment, which, when ligated, yields a third vector product. The host organism that is permissive for replication of the vector is transformed with the third vector product to facilitate its replication.

The target organism to be sequentially cloned is then transformed with the third vector product, and circular integration of this vector into the chromosomal DNA segment to be cloned is allowed to occur. The resulting recombinant chromosomal DNA segment includes two homologous DNA segments, one at either end of the vector segment. The resulting circularly integrated chromosomal DNA is then cleaved with the retrorestriction enzyme specific for the third restriction site, and the termini of DNA fragments in the digest are rendered blunt-ended by treatment with a suitable agent. The fragments are then circularized by ligation at Iow DNA concentration to form a fourth vector product. The permissive host is transformed with the fourth vector product to allow its selective replication.

A greater length of the chromosome can be sequentially cloned. This can be accomplished by cleaving the fourth vector product with the restriction enzyme specific for the first restriction site to produce a DNA segment containing the vector and a short segment of DNA that is homologous to 
a portion of the DNA segment excised with the retrorestriction enzyme. Upon circularization by ligation this becomes the fifth vector product.

Next, the host organism that is permissive for the replication of the vector is transformed with the fifth vector product to facilitate its replication. The organism to be sequentially cloned is then transformed with the fifth vector product, and circular integration is allowed to occur. The zhromosomal DNA containing the vector in once again cleaved with the second restriction enzyme, the site for which remains after cleavage with the retrorestriction enzyme in the prior step.

The process may be repeated to sequentially clone further along the length of the chromosome, and under favorable circumstances to sequentially clone the entire length of the chromosome.

An advantage of the present invention is that it can be used to sequentially clone chromosomal DNA without an extensive knowledge of the DNA to be sequentialily cloned.

A further advantage of the present invention is that it can be used to sequentially clone entire chromosomes.

A further advantage is that the present invention provides an immediate map of the chromosome in terms of overlapping cloned segments characterized by several restriction sites. 
A still further advantage of the present invention is that it provides cloned chroinosomal DNA that can be subjected to nucleotide sequence analysis or to genetic modification.

For a better understanding of the present invention reference is made to the following description and figures, the scope of which is pointed out in the appended claims.

\section{BRIEF DESCRIPTION OF THE DRAWINGS}

Figure 1 illustrates the mechanism of circular integration.

Figure 2 illustrates a preferred method of the present invention for the sequential cloning of chromosomal DNA.

Figure 3 illustrates overlapping segments of cloned DNA produced by the sequential cloning process illustrated in Figure 2 .

Figure 4 illustrates the formation of the pWG5 vector.

Figure 5 illustrates two double-stranded oligonucleotides that can be used in the formation of vectors. The oligonucleotide illustrated in $A$ was used in the formation of pWG5.

Figure 6 illustrates a segment of $\underline{S}$. pneumoniae sequentially cloned using the method of the present invention.

Figure 7 illustrates a Southern blot confirmation of a partial chromosomal map of $\mathbf{s}$. pneumoniae obtained with the present invention. 
Figure 8 illustrates the method of the present invention as used for the sequential cloning of part of the S. pneumoniae chromosome.

Figure 9 illustrates vectors for use with the method of the present invention having initial chromosomal segments inserted in opposite orientations.

\section{DETAILED DESCRIPTION OF THE PRESENT INVENTION}

For purposes of understanding the present invention, the following terms are defined:

Homologous DNA - Segments of DNA having the same base sequence.

Non-Homologous DNA - Segments of DNA which do not have the same base sequence.

Walking Along the Chromosome - The extension of cloned DNA from a particular point or segment in the chromosome. The term has been used to describe the screening of a library of random genomic clones by DNA hybridization to find clones overlapping the initially cloned segment, see, Bender et al., "Chromosomal walking and jumping" to isolate DNA from the Ace and rosy loci and the bithorax complex in Drosophila melanogaster", J. Mol. Biol., 168, 17-33 (1983). In the method of the present invention, however, a vector steps along the chromosome as part of the cloning process itself and overlapping clones are produced. The present invention can be used to extend a region of cloned sequence and, in theory, to sequentially ćlone an entire genome. 
Circular Integration - The sequential cloning approach used in the present invention is based on integration into the chromosome of circular structures composed of DNA segments that are respectively homologous and heterologous to the chromosome. Such integration at various times has been called ectopic integration, see, stassi et al., "cloning of chromosomal genes in Streptococcus pneumoniae", Proc. Natl. Acad. Sci. USA, 78, 7028-7032 (1981); and Mannarelli et al., "Ectopic integration of chromosomal genes in streptococcus pneumoniae, J. Bacteriol.", 160, 867-873 (1984); additive transformation, see, claverys et al., "Identification of Streptococcus pneumoniae mismatch repair genes by an additive transformation approach", Mol. Gen. Genet., 196, 91-96 (1984); and duplicative insertion, see, Morrison et al., "Isolation of transformation-deficient streptococcus pneumoniae mutants defective in control of competence, using insertion-duplication mutagenesis with the erythromycin resistance determinant of pAMB1", J. Bacteriol., 159, 870876 (1984). Here we shall refer to it as circular integration. Circular integration appears to result from the interaction by circular synapsis of a circular donor molecule with the recipient cell chromosome so that a single-strand crossover integrates the entire circular structure in an additive manner into the chromosome; for a review of this process see Lacks, S.A., "Mechanisms of 
genetic recombination in gram-positive bacteria", Genetic Recombination, Am. Soc. Microbiol., Washington, D.C., p. 4385 (1988). Prior to integration, the double stranded circular donor molecule is broken within the homologous portion during DNA uptake by the cell and converted to a single stranded donor molecule.

Circular integration only occurs if the circular donor molecule is not able to replicate autonomously in the recipient cell. If the circular donor molecule can replicate within the recipient cell then the circular donor molecule's interaction with the chromosome by circular synapsis, i.e. the pairing of homologous DNA, is merely a transient step that facilitates plasmid establishment, see, Lopez et al., "Facilitation of plasmid transfer in streptococcus pneumoniae by chromosomal homology", J. Bacteriol., 150, 692-701 (1982); and Canosi et al., "Plasmid transformation in Bacillus subtilis:effects of insertion of Bacillus subtilis DNA into plasmid pC194", Mol. Gen. Genet., 181, 433-440 (1981).

Circular integration is illustrated in Figure 1. A circular double stranded plasmid is created by ligating homologous and non-homologous DNA restriction fragments to form the double stranded circular DNA plasmid illustrated in Figure 1(a). The plasmid is introduced into the bacterium to be transformed. The plasmid becomes single stranded during the transformation process. Circular synapsis of a 
single stranded donor fragment occurs at the portion of the chromosome homologous to the donor DNA as illustrated in Figure 1(b). A single strand crossover integrates the circular donor strand into the chromosome as illustrated in Figure 1(c).

If the circular DNA structure has no origin of replication suitable for replication in a given host, it cannot replicate within that host. Accordingly, the circular donor DNA remains associated "ith the chromosiome until 1) the donor DNA is degraded or 2) a single-strand recombination event occurs with the chromosomal homolog to the donor DNA, and the donor DNA is inserted into the chromosome by circular integration. In this second situation, called circular integration, replication of the chromosome converts the integrated single strand segment into a duplex form so that the heterologous donor segment is located between duplicated homologous segments. Thus, the product of circular integration contains a direct repeat of the homologous segment flanking the heterologous DNA as is illustrated in Figure 1 (d).

The method of the invention includes the circular integration of a circular DNA vector product into a chromosome. The circular DNA vector product may, for example, contain a plasmid or cosmid vector. The circular vector product is composed of segments of DNA homologous and heterologous to the chromosomal DNA. During circular 
integration, the circular vector product is broken and the entire vector product is taken into the chromosome of the recipient host bacterium. The heterologous DNA corresponding to the vector is not able to replicate within the recipient bacterium, called a non-permissive bxcterium for the vector. The heterologous DNA vector can, however, replicate in a bacterium permissive for replication of the vector, called a permissive bacterium for this vector. The process by which a bactrrium takes up plasmids is referred to as transformation.

The sequential cloning method is carried out by circular integration of a vector containing a small segment of DNA homologous to the chromosome followed by excision of a plasmid containing a much larger segment of chromosomal DNA. That recombinant plasmid is subsequently reduced to contain only a small segment of chromosomal DNA located at the other end of the large insert, which is then used for the next circular integration step. The process of the present invention depends on the judicious use of restriction sites in a vector constructed for this purpose. The method should be suitable for sequentially cloning chromosomal DNA in any organism giving adequate frequencies of circular integration. Examples of suitable organisms include the transformable bacteria and yeasts. The process of the present invention includes the use of a vector ( $V$ ). The vector (V) must be designed to contain 
at least three adjacent restriction endonuclease recognition sites, which are not present anywhere else in the vector. The first restriction site (D) must be such that the distance between $D$ restriction sites in the chromosome will be small. Suitable D restriction enzymes include DpnI, Sau3A1, and other endonucleases with 4-bp recognition sites, including those listed in Roberts, "Restriction enzymes and their isoschizomers", Nucleic Acids Res., r271-r313 (1988). Preferably, the distance between D restriction sites will be on the order of $250 \mathrm{bp}$. The D site will preferably be specific for a 4-bp cutter. The second site (E) is a site which will preferably allow for cloning of large chromosomal DNA fragments. Suitable E restriction site enzymes include EcoRI, SphI, FspI, and other endonucleases with 6-bp recognition sites, including those listed in Roberts, "Restriction enzymes and their isoschizomers", Nucleic Acids Res., 16, r271-r313 (1988). Preferably, E sites will average from $3 \mathrm{~kb}$ to $15 \mathrm{~kb}$ in length and the E site will be specific for a 6-bp cutter. The third site must cleave the DNA outside of the recognition sequence, so that the adjacent restriction site is preserved. In other words, the restriction enzyme for the F site must have the property of retrorestriction and therefore be a class IIS restriction enzyme. Suitable F site enzymes include BspMI, Tth111II and other class IIS endonucleases found in the listing of szybalski, et al., "Class-IIS restriction enzymes - a 
review", Gene, 100, 13-26 (1991). The third site is preferably specific for a 6-bp cutter. Most preferably, the E site is also specific for a retrorestriction enzyme.

The vector (V) can replicate autonomously only in the permissive host (P), and not in the nonpermissive host (target) containing the chromosome to be sequentially cloned. The vector must also carry a marker(s) expressible in both hosts to allow selection of transformants. Suitable markers are well known to those skilled in the art and include, for example, genes conferring resistance to certain antibiotics.

The process of the present invention begins with the isolation of a fragment of DNA $\left(s_{1}\right)$ homologous to a segment of chromosomal DNA. The homologous fragment $\left(s_{1}\right)$ is preferably obtained either from a chromosomal segment previously cloned for another purpose or a segment taken randomly from a $D$ enzyme restriction digest of chromosomal DNA. The homologous fragment $\left(S_{1}\right)$ is ligated into the $D$ restriction site of the vector to form a circular plasmid (Figs. 2(a) and (b)). The resulting plasmid is then used to transform the non-permissive target host bacterium. The homologous segment $\left(S_{1}\right)$ directs the vector insertion into the chromosome of the target bacterium (Fig. 2(c)). The circular mode of integration results in a duplication of the segment $\left(S_{1}\right)$ bracketing the vector (Fig. $2(d)$ ). In other words, the chromosomal DNA of the target bacterium now 
includes two $s_{1}$ segments at either end of the inserted vector DNA.

The chromosomal DNA is then treated with restriction enzyme $E$ which cleaves the chromosomal DNA at one end of the inserted vector and at a point further down the chromosome. In other words, the chromosomal DNA is cleaved to produce a segment including the vector DNA (V) and a section of chromosomal DNA $\left(S_{1}, L\right.$ and $\left.S_{2}\right)$. The excised DNA is ligated to form the circular plasmid illustrated in Figure $2(e)$. The circular plasmid is then used to transform the permissive host bacterium (P) and produce recombinant plasmids in which a substantial segment $\left(S_{1}, L_{1}\right.$ and $\left.S_{2}\right)$ of the target chromosome is cloned. The resulting plasmid can be sequenced and will include the sequence $S_{1}-I_{1}-S_{2}$.

To walk further down the chromosome, the previous plasmid [Fig. 2(e)] is cut with restriction enzyme $D$ and ligated to form the plasmid illustrated in Figure $2(f)$. In general there will be multiple $D$ sites in the cloned $E$ fragment. Such cutting will therefore remove most of the insert and leave only a distal segment between the site closest to the end of the insert at the E site (i.e., $s_{1}$ and $L_{1}$ will be excised). Ligation produces a reduced plasmid carrying the $s_{2}$ fragment illustrated in Figure $2(f)$. The $S_{2}$ plasmid illustrated in Figure $2(f)$ is then used to transform the target bacterium. Transformation of the target bacterium again inserts the vector into the target 
organism chromosome (by the circular integration mechanism previously described), but further downstream at the site of homology with $S_{2}$ as illustrated in Figures $2(g)$ and $2(h)$. The circular integration process results in a duplication of the $S_{2}$ segment bracketing the vector as shown in Figure $2(g)$, i.e. the target organism chromosomal DNA now includes two $s_{2}$ segments at either end of the inserted vector.

A retrorestriction enzyme (enzyme F) is used to cut out a large fragment of the target chromosome containing the vector. Enzyme $F$ preferably has a 6-bp recognition sequence. Retrorestriction enzyme $F$ cleaves upstreain of its recognition sequence in the vector so that the adjacent $\mathbf{E}$ site is retained. The use of the retrorestriction enzyme can result in the production of a $5^{\prime}$ or $3^{\prime}$ overhang. The overhang is treated with an agent to facilitate ligation by producing biunt ends. Suitable agents for 5 ' overhangs include polymerases such as, for example, the klenow fragment and T4 DNA polymerase. Suitable agents for $3^{\prime}$ overhangs include mung bean nuclease and $S 1$ nuclease. The restriction enzyme $F$ fragment ( $F$ fragment) is ligated to form the circular plasmid illustrated in Figure 2(i). The $F$ fragment contains a second large target chromosomal fragment which includes chromosomal DNA segments $S_{2}, L_{2}$ and $S_{3}$. The $F$ fragment plasmid transforms the permissive host to yield clones of the $F$ fragment. The F fragment plasmid can be sequenced and will include $S_{2}-L_{2}-S_{3}$. 
The $\mathrm{F}$ fragment plasmid is treated with restriction enzyme $D$ which removes $S_{2}$ and $L_{2} \cdot S_{2}$ and $L_{2}$ are removed so that the $S_{3}$ segment can be circularly integrated into the vector. The resulting Enzyme D digestion product is ligated to yield the $s_{3}$ containing vector illustrated in Figure $2(j)$. The $s_{3}$ containing vector is integrated into the target bacterium chromosome by circular integration of the vector as illustrated in Figures $2(\mathrm{k})$ and $2(1)$. The circular i-tegration process results in a duplication of the $\mathrm{S}_{3}$ segment bracketing the vector (Figure 2(1). Therefore, the target organism chromosomal DNA now includes two $\mathrm{S}_{3}$ segments at either end of the inserted vector.

Cutting the circularly integrated chromosomal DNA of Figuse 2(1) with restriction enzyme $\mathrm{E}$ is repeated, followed by the other steps, and after the next integration, cutting with restriction enzyme $F$ and subsequent steps are repeated as illustrated in Figure 2. In this manner alternate segments are cloned down the chromosome as restriction enzyme $E$ and restriction enzyme $F$ fragments. The process of this invention results in the production of successive clones of large chromosomal DNA segments. The homologous DNA of the clones overlap as illustrated in Figure 3 . In Figure 3, S represents homologous overlapping chromosomal DNA, I represents non-overlapping chromosomal DNA and D, E and $F$ represent cutting sites for different restriction 
enzymes. The process of the present invention is particularly useful for genome mapping and sequencing.

The present invention does not require knowledge of the chromosomal sequence to be cloned. All that is required to start the process is a segment of DNA having appropriate restriction sites at each end. The fragment can, for example, be produced by treating the chromosomal DNA with an appropriate restriction enzyme.

If the initial chromosomal segment $\left(S_{1}\right)$ is originally inserted in the opposite orientation in the vector (see Fig. 9), then sequential cloning will proceed along the chromosome in the opposite direction. In practice, inserts will be obtained in both orientations. The direction of insertion is determined, prior to the start of the sequential cloning process, by cutting with restriction enzymes for $E$ and $G, a$ unique site that is off center in the insert. In one orientation the fragment from $E$ to $G$ is short, in the other orientation, it ia long. Since vectors with inserts in opposite orientations will proceed in opposite directions along the chromosome, by starting with one chromosomal fragment the entire chromosome can, in principle, be cloned. Five overlapping clones in one direction are illustrated in Figure 3. A physical and genetic map of the chromosome can thus be determined, and clones can be sequenced using conventional sequencing 
techniques. Various considerations which should be taken into account are considered in the following Example.

Example 1: sequential cloning of part of the 8 . Pneumoniae in E. coli.

Genome Using Vectors That can Replicate

\section{a) Construction of vectors}

Vectors that can replicate in E. coli but not in S. pneumoniae and that carry a marker expressed in both species were constructed by combining segments of pBR322 and pJS3 as shown in Fig. 4. Experimental manipulations were carried out according to the suppliers of the restriction enzymes or to Maniatis, et al., Molecular Cloning, $\underline{A}$ Laboratory Manual, Cold spring Harbor Lab., Cold Spring Harbor, NY (1982). The marker used was the cat gene from the staphylococcal plasmid pc194, the sequence of which is known, see, Horinouchi et al., "Nucleotide sequence and functional map of pc194, a plasmid that specifies inducible chloramphenicol resistance", J. Bacteriol., 150, 815-825 (1982); and which confers $\mathrm{Cm}^{R}$ (chloramphenicol resistance)

to both E. coli, see, Lacks et al., "Identification and analysis of genes for tetracycline resistance and replication functions in the broad-host-range plasmid pLS1", J. Mol. Biol., 192, 753-765 (1986), and S. pneumoniae, see, Ballester et al., "Selective advantage of deletions enhancing chloramphenicol acetyltransferase gene expression in streptococcus pneumoniae plasmids", Gene, 41, 153-163 (1986). The source of this marker was a 1.1-kb DpnII fragment of pJS3, which carries an active promoter preceding 
the cat gene, as reported by Ballester et al., "Selective advantage of deletions enhancing chloramphenicol acetyltransferase gene expression in streptococcus pneumoniae plasmids", Gene, 41, 153-163 (1986); and a transcription terminator after it, as reported by de la Campa et al., "Proteins encoded by the DpnII restriction gene cassette: two methylases and an endonuclease", J. Mol. Biol., 196, 457-469 (1987). The E. coli replicon was a 1.4kb fragment, bp 1668 to 3042 , described by Balbas et al., "Plasmid vector PBR322 and its special-purpose derivatives-a review", Gene, 50, 3-40 (1986), of pBR322 grown in E. coli strain GM33, which was excised by DpnII. This fragment retains the replication functions, but it lacks the EcoRI and BspMI sites of pBR322 and all DpnII sites except those at its termini. Ligation of the two fragments and transformation of E. coli gave pWG10 (see Fig. 4). Surprisingly, the 1.4-kb DpnII fragment of pBR322 was sufficient to enable replication of the vector plasmid. It had previously been believed that the pBR322 promoter at bp 3124-3096 was necessary for plasmid replication, see, Balbas et al., "Plasmid vector pBR322 and its special-purpose derivatives--a review", Gene, 50, 3-40 (1986). Perhaps a sequence in the cat segment of the vector took its place. One possibility is the sequence 5'-TTGAtt-

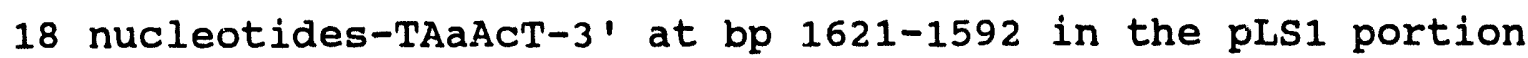
of that segment, see, Lacks et al., "Identification and 
analysis of genes for tetracycline resistance and replication functions in the broad-host-range plasmid pLsi", J. Mol. Biol., 192, 753-765 (1986); which agrees fairly well (upper case symbols) with the consensus E. coli promoter, see, Rosenberg et al., "Regulatory sequences involved in the promotion and termination of RNA transcription", Ann. Rev. Genet., 13, 319-353 (1979).

One DpnI site was removed from pWG10 by partial cleavage with Sau3PT, treatment with S1 nuclease, and ligation to give pWGI (Fig. 4). Sequence analysis showed that 51 nuclease digestion extended past the overhanging strand at the restriction site. The s1 nuclease digestion resulted in a deletion of bp 3030-3041 in the pBR322 segment and a deletion of the cat segment from bp 1495-1515 (in the pLS1 portion). The single BspMI site in pWG1, which is located in the cat segment but past the end of the cat structural gene, was removed by BspMI cleavage and limited digestion with BAL-31 nuclease. This treatment deleted bp 1954-1982 in the pc194 numbering. The resulting plasmid, pWG3, had only a single DpnI site and no BspMI, ECoRI, FspI or SphI sites.

Into the unique DpnI site of pWG3, we inserted the two double-stranded synthetic oligonucleotides shown boldface in Fig. 5. The oligonucleotide strands were synthesized by the phosphoramidite method on a systec Microsyn 1450 DNA synthesizer. The insert of Fig. 5B 
produces the DpnI-ECoRI-BspMI sequence described above. The insert of Fig. 5A, which gives the sequence DpnI-SphI-FspIBspMI, contains two 6-bp recognition sites restorable after cleavage with BspMI. The advantage of such multiple sites will be discussed below. The plasmid with the DpnI-sphIFspI-BspMI insert, pWG5, was used to demonstrate sequential cloning.

\section{b) sequential cloning of the s. pneumoniae genome}

Lacks and Greenberg, "Sequential cloning by a Vector Walking Along the Chromosome", Gene, 104, 11-17 (1991) and references therein. The mal locus of $\underline{S}$. pneumoniae, which controls utilization of the sugar maltose, was previously mapped genetically, see, Lacks, S., "Genetic regulation of maltosaccharide utilization in pneumococcus", Genetics, 60 , 685-706 (1968). A 3.5-kb segment containing the malM gene and the start of the following malp gene was cloned and sequenced in pLS70, see, Lacks et al., "Identification of base mismatches recognized by the heteroduplex-DNA-repair system of streptococcus pneumoniae", Cel1, 31, 327-336 (1982). For a starting point in the s. pneumoniae chromosome, a 373-bp sauusAI fragment from within malM (labeled S1 in Fig. 6) was inserted into pWG5, oriented with its transcriptional polarity the same as cat, to give plasmid ps1. The psi plasmid, propagated in E. coli, 
transformed S. pneumoniae to yield $6000 \mathrm{~cm}^{R}$ clones $/ \mathrm{ml}$. These clones, as expected, contained no plasmids. DNA from one clone was digested with SphI, ligated to circularize the fragments, and used to transform E. coli. The resulting plasmid ( $p L 1$ ), which was present in all $6 \mathrm{~cm}^{\mathrm{R}}$ transformants that were obtained, contained a pneumococcal chromosomal DNA segment of $1.7 \mathrm{~kb}$. The pL1 plasmid was reduced by digestion with Sau3AI to contain only a $0.2-\mathrm{kb}$ segment ( $\mathrm{S} 2$ in Fig. 6 ). Subsequent transformation of $\underline{s}$. pneumoniae with ps2 gave $1600 \mathrm{~cm}^{\mathrm{R}}$ clones/ml. Chromosomal DNA from one clone was digested with BspMI. BspMI produced a 4-base 5'overhang that was filled in by DNA synthesis using the Klenow fragment of DNA polymerase $I$, which is also known as PolIk. The BspMI-treated fragment was circularized by ligation and used to transform E. coli. Two of three $\mathrm{cm}^{\mathrm{R}}$ transformants contained a plasmid with a 1.3-kb insert, L2. The third appeared to contain ps2, which could have been produced by BspMI excision if the originally integrated pS1 were duplicated as a consequence of the repeated sequences bordering it, see, Vasseghi et al., "Amplification of a chimeric plasmid carrying an erythromycin-resistance determinant introduced into the genome of streptococcus pneumoniae", Gene, 21, 285-292 (1983). Plasmid pL2 was reduced by digestion with SauAI to give pS3, a $2.8-\mathrm{kb}$ plasmid. As expected, pS3 retained the SphI site. 
Transformation of $\underline{S}$. pneumoniae with ps3 gave $1000 \mathrm{Cm}^{\mathrm{R}}$ clones/ml. When DNA from one of them was digested with SphI, circularized, and used to transform E. coli, 11 $\mathrm{Cm}^{\mathrm{R}}$ transformants tested all contained only the original ps3 plasmid. Perhaps the next SphI segment could not be cloned, either because it was too large or because it contained a gene toxic in a high copy number plasmid. However, resort was made to the FspI site in the vector. When the DNA containing integrated ps3 was cut with FspI, circularized, and used to transform E. coli, 9 of $15 \mathrm{~cm}^{R}$ transformants contained pL3, an $8.4-\mathrm{kb}$ plasmid. The remaining $6 \mathrm{~cm}^{R}$ transformants contained ps3.

\section{c) Restriction mapping of sequentially} cloned DNA Restriction analysis of $\mathrm{pL} 1, \mathrm{pL} 2$, and $\mathrm{pL} 3$ gave the map shown in Fig. 6 for the region downstream from malM. The positions of the $S$ and $L$ segments are indicated on the map. The map is consistent with sequential cloning expectations in that no SphI sites occur within L1, no BspMI sites within L2, and no FspI (or SphI) sites occur within L3.

One step of sequential cloning was taken in the opposite direction with a vector containing an EcoRI site and the $\mathrm{SI}$ segment. In this case the vector contained oligonucleotide B of Fig. 5, and the S1 segment was oriented in the vector with its transcriptional polarity opposite to cat. Otherwise experimental conditions were similar to the 
sequential cloning with pWG5. This step extended the mal region map $0.6 \mathrm{~kb}$ to the left, as shown in Fig. 6 .

\section{blotting}

d) Confirmation of chromosomal map by southern The accuracy of the sequential cloning procedure was tested by cutting the chromosomal DNA of $\underline{S_{\text {. }}}$ pneumoniae with various restriction enzymes and testing for hybridization with probes made from the cloned DNA (pS1 and pL2). Results in Fig. 7 show that pS1 hybridized to a 5-kb EcoRI fragment and to a 1.4-kb KpnI fragment as expected from Fig. 6. The 5-kb KpnI fragment detected by psi presumably extends to the left of the KpnI site in malM (Fig. 6). Both ps1 and pL2 hybridized to a BglII fragment of identical size, estimated to be $15 \mathrm{~kb}$. The SphI fragment to which pSI hybridized is estimated to be approximately 30 kb. For pL2, hybridization with 5-kb KpnI, 1.5- and 1.8-kb $\underline{P s}+I$, and $7.5-\mathrm{kb}$ BamHI-BqlII fragments corresponded to the restriction map. Only the $7.8-\mathrm{kb}$ FspI fragment to which pL2 hybridized appeared significantly larger than the $7.2-\mathrm{kb}$ expectation. Perinaps a small deletion occurred in the plasmid taken as the pL3 prototype. Inasmuch as the fragments from the chromosome are generally the size predicted from the cloned segments, we conclude that the cloning was indeed sequential according to the scheme outlined in Fig. 8 . 


\section{the present invention \\ e) Further considerations in using the method of Occasionally, the alternate 6-bp recognition}

site will occur in the small fragment used for circular integration. For example, in the EcoRI cycle after a BspMI cycle, as depicted in Fig. 8 , if an EcoRI site occurs within the small segment $\left(S_{3}\right)$, the cloning cannot be extended further. This situation is detectable by the failure to clone a larger fragment. Sequential cloning can be continued, however, by the use of a vector having a different restriction site.

Figure 4 illustrates a more sophisticated vector, pWG5, having two 6-bp recognition sites, SphI and FspI. The combined limit of which should average 24 cycles. Changing a single $G: C$ bp to $C: G$ in the fourth position from the end of the oligonucleotide shown in Fig. $5 \mathrm{~A}$ would produce a Tth111II site, 5'-TGYTTG-3', and the single Tth111II site in the vector (bp 1921-1926 in pBR322) could be eliminated. Since Tth11III, like BspMI, is a retrorestriction enzyme that will cleave in back of the FspI site, these two enzymes could substitute for each other indefinitely. However, since TthlllII gives a 3 ' overhang, fragments by it will be treated with an agent such as mung bean nuclease, rather than PolIk, to make blunt ends. Thus, as many as 48 cloning cycles might be achieved without interruption. 
In other circumstances, a fragment may not be cloned because it is either physically too large or because it contains elements inimical to the host plasmid or cell. Several modifications of the procedure can alleviate such problems. For example, when cloning the genome of a species like S. pneumoniae that contains only $40 \%$ G+C in its DNA, it would be preferable to use vectors with restriction sites richer in $A+T$ to obtain $L$ segments of smaller average size.

It has been reported that chromosomal DNA

fragments from $\underline{s}$. pneumoniae containing strong promoters are not readily clonable in E. coli plasmids, see, Chen, et al., "Cloning of streptococcus pneumoniae DNA fragments in Escherichia coli requires vectors protected by strong transcriptional terminators", Gene, 55, 179-187 (1987). This problem may, however, be circumvented by the introduction of transcription terminators bracketing the insert in the $\underline{E}$. coli vector, as reported by chen et al. (1987) supra.

Furthermore, foreign protein products expressed in E. coli may be toxic, particularly when their genes are cloned in high copy number. For this reason it may be preferable to use a low copy vector for sequential cloning. Suitable low copy vectors include pSC101 or pWG5, itself, when grown in an E. coli host carrying the pen mutation. Another situation that may be encountered in the sequential cloning of bacterial chromosomes is that 
during the vector integration phasc of the cycle, insertion of the vector into the target chromosome could interrupt an essential gene. This would typically occur if the small

homologous (S) fragment lay entirely within the gene. This situation can be circumvented by using a larger homologous fragment. The larger fragment could be obtained, for example, by incompletely cleaving the previous $L$ insert with DpnI, so that the $S$ segment is not entirely within the essential gene.

integration in bacteria occur in yeast cells after

transformation with circularly structured DNA. Although, in this case the interaction apparently occurs between doublestranded donor DNA molecules and the chromosome, as reported by orr-Weaver and szostak, "Yeast recombination: the association between double-strand gap repair and crossingover", Proc. Natl. Acad. Sci. USA, 80, 4417-4421 (1983). When a donor molecule that is composed of a vector unable to replicate in yeast joined at both ends to a segment of DNA homologous to the yeast genome transforms the yeast cell, it is circularly integrated into the position of homology in the yeast chromosome, see, Hinnen et al., "Transformation of yeast", Proc. Nat1. Acad. Sci. USA, 75, 1929-1933 (1978). It is possible, also in yeasts, to subsequently excise a larger fragment of the chromosome and clone it in a host 
that supports replication of the vector, see, stiles et al., "DNA sequence of a mutation in the leader region of the yeast iso-1-cytochrome c mRNA", Cell, 25, 277-284 (1981). Therefore, the procedure described for sequential cloning of a bacterial genome should be applicable also to yeast chromosomes.

Some difficulties encountered in sequentially cloning bacterial DNA may not be troublesome in yeast. In diploid cells, inter:uption of one copy of an essential gene may be inconsequential. Differences in protein translation between eukaryotes and prokaryotes may diminish the problem of toxic gene expression in the bacterial host. However, other difficulties may arise that are characteristic of eukaryotic DNA, for example, in extending the cloning through sequences of repetitive DNA.

The sequential cloning approach cannot be directly applied to chromosomes in mammalian cells because DNA that is introduced into mammalian cells is generally inserted indiscriminately into chromosomes rather than integrated at positions of homology, see, Robins et al., "Transforming DNA integrates into the host chromosome", Cel1, 23, 29-39 (1981). However, large segments (up to 800 $\mathrm{kb}$ ) of human DNA have been cloned as artificial chromosomes in yeast, see, Little et al., "Yeast artificial chromosomes with 200- to 800-kilobase inserts of human DNA containing HLA, V, 5S, and Xq24-Xq28 sequences", Proc. Natl. Acad. Sci. 


$$
-30-
$$

USA, 86, 1598-1602 (1989); and human or other mammalian DNA may be subject to sequential cloning in this form. While there have been described what are the presently contemplated preferred embodiments of the invention, those skilled in the art will realize that various changes and modifications may be made to the invention without departing from the spirit of the invention, and it is intended to claim all such changes and modifications a: falling within the scope of the present invention. 


\section{ABSTRACT \\ SEQUENTIAL CLONING OF CHROMOSOMES}

A method for sequential cloning of chromosomal DNA and chromosomal DNA cloned by this method are disclosed. The method includes the selection of a target organism having a segment of chromosomal DNA to be ser:entially cloned. A first DNA segment homologous to the chromosomal DNA to be sequentially cloned is isolated. The first segment has a first restriction enzyme site on either side. A first vector product is formed by ligating the homologous segment into a suitably designed vector. The first vector product is circularly integrated into the target organism's chromosomal DNA. The resulting integrated chromosomal DNA segment includes the homologous DNA segment at either end of the integrated vector segment. The integrated chromosomal DNA is cleaved with a second restriction enzyme and ligated to form a vector-containing plasmid, which is replicated in a host organism. The replicated plasmid is then cleaved with the first restriction enzyme. Next, a DNA segment containing the vector and a segment of DNA homologous to a distal portion of the previously isolated DNA segment is isolated. This segment is then ligated to form a plasmid which is replicated within a suitable host. This plasmid is 
then circularly integrated into the target chromosomal DNA. The chromosomal DNA containing the circularly integrated vector is treated with a third, retrorestriction (class IIS) enzyme. The cleaved DNA is ligated to give a plasmid that is used to transform a host permissive for replication of its vector. The sequential cloning process continues by repeated cycles of circular integration and excision. The excision is carried out alternately with the second and third enzymes. 

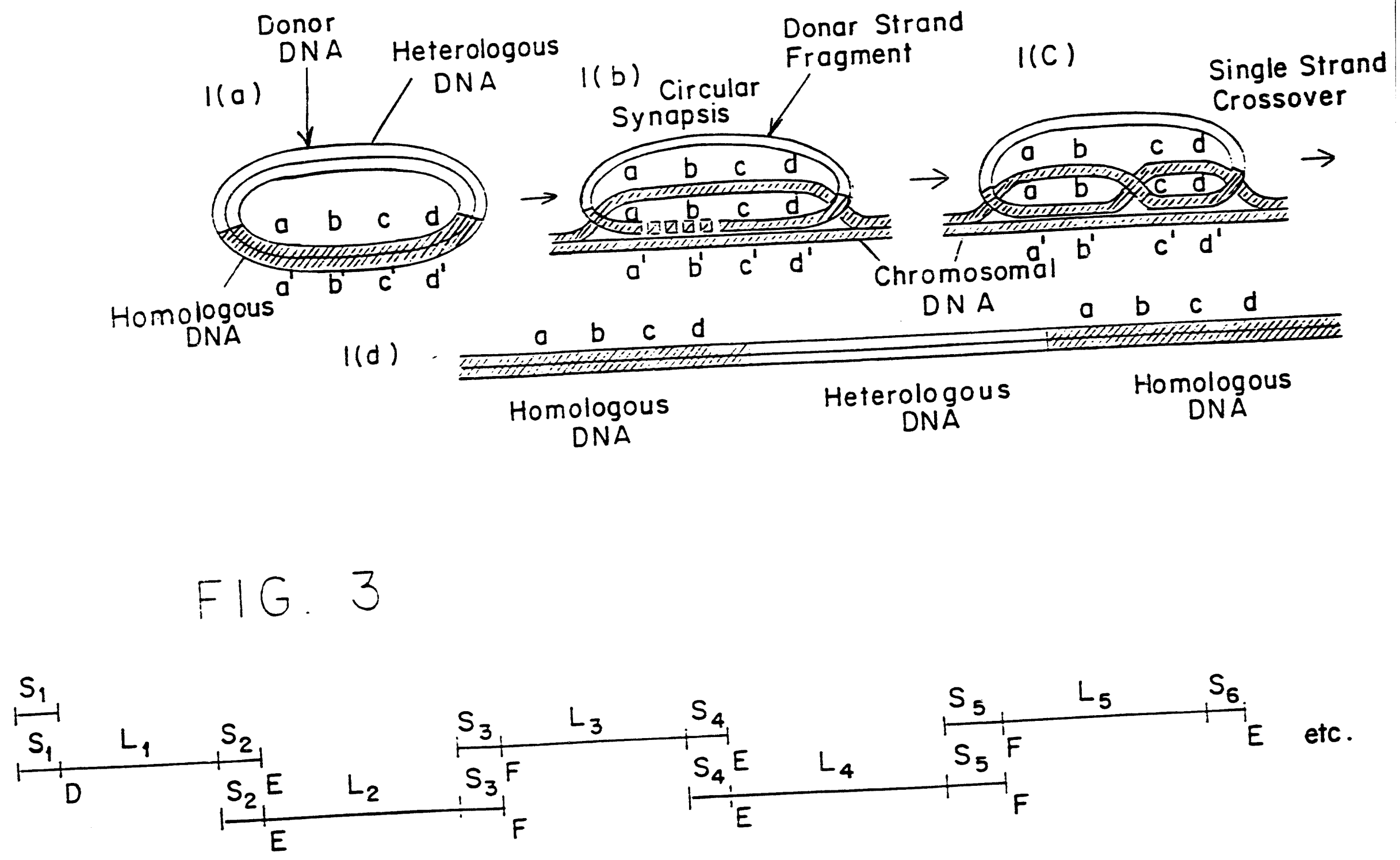


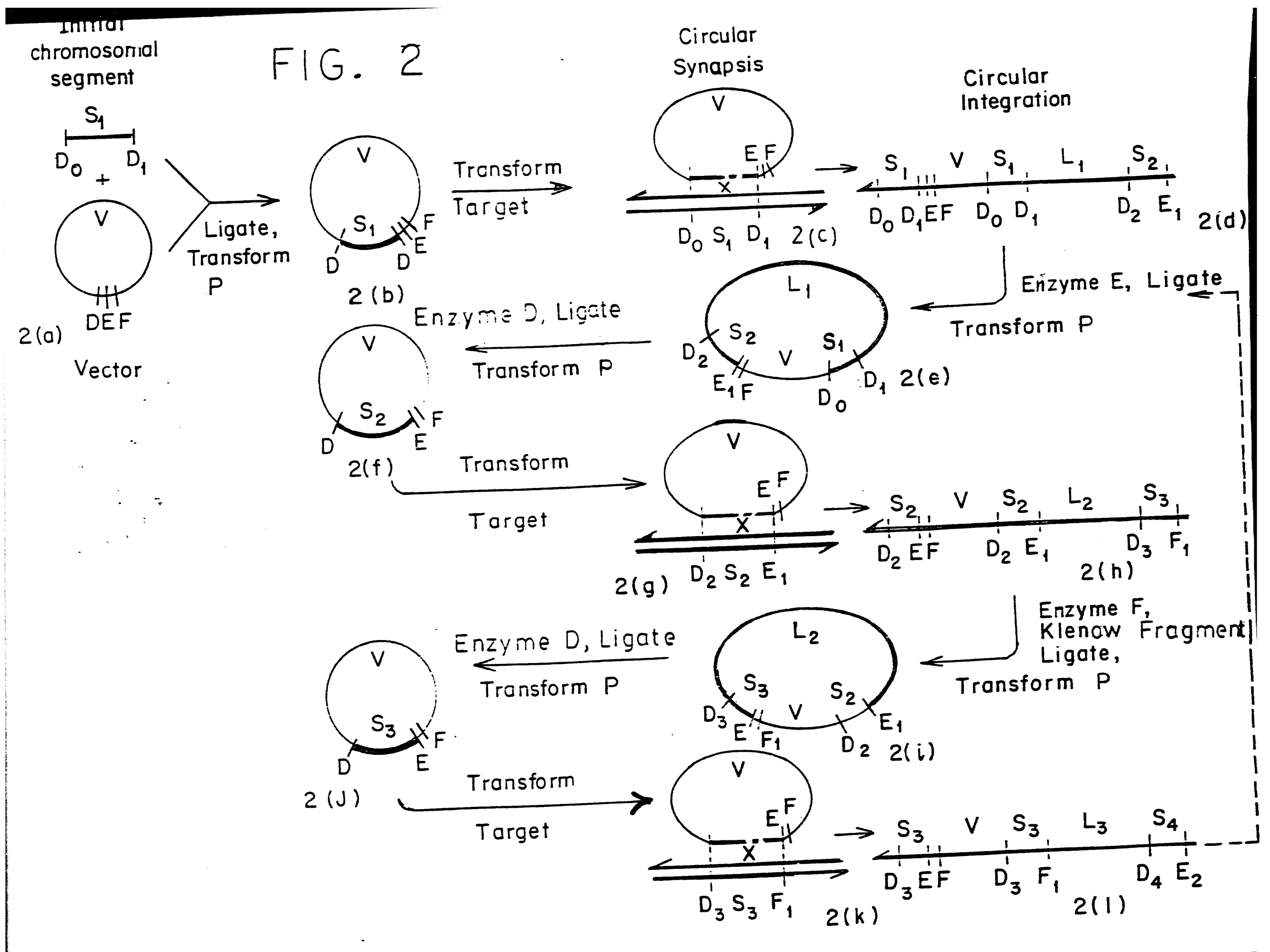


FIG. 4

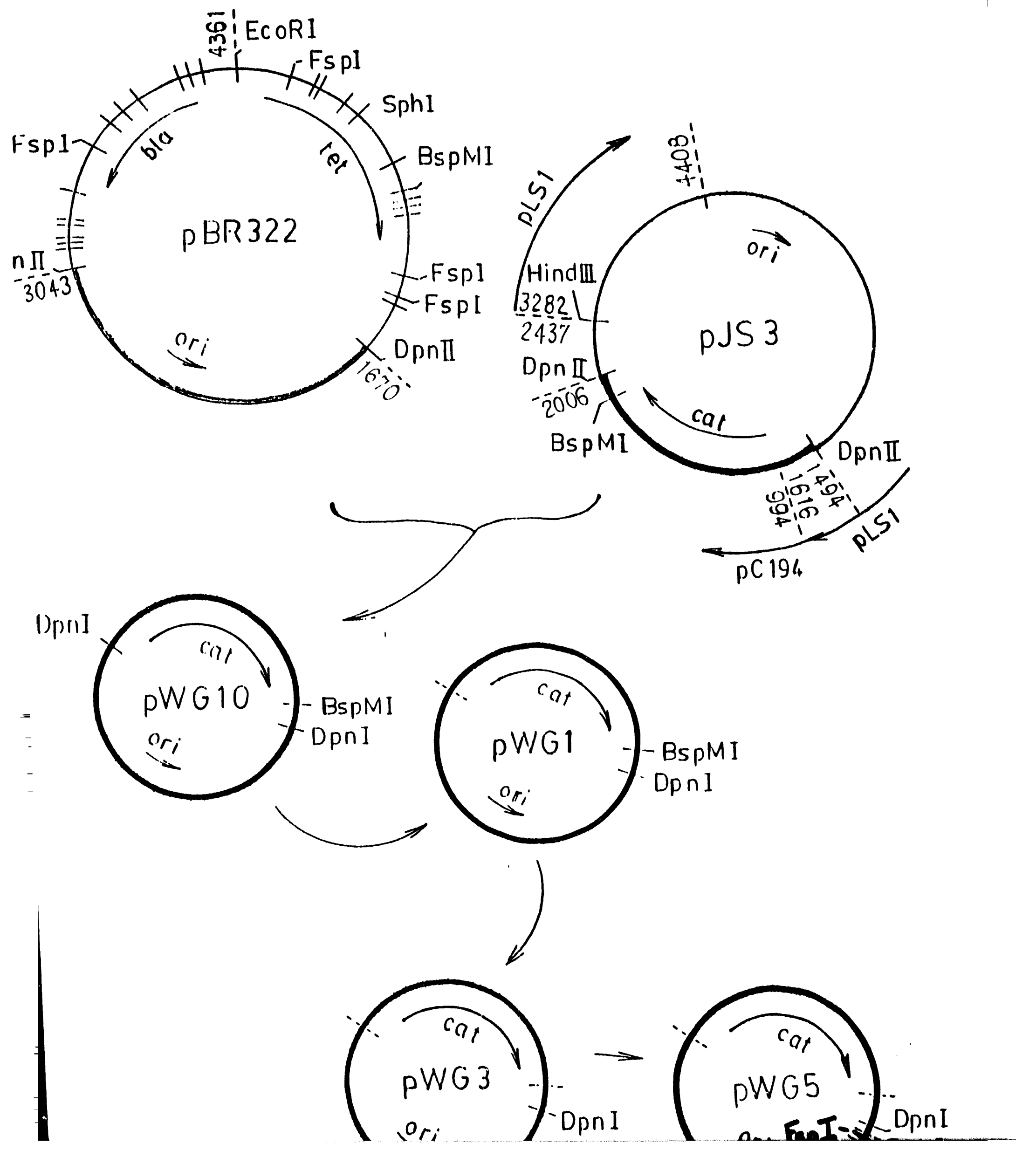


FIG. 5

Fsp I

$m$

5-GATCGCATG C G CA G GT G G T T G T C-3

$3^{\prime}-$ C TAG CG TACGCG T C CACCAACA $A \mathrm{~m}^{-} 5^{\prime}$

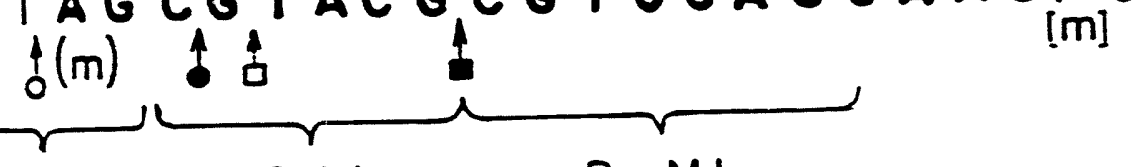

Dpnl Sphl BspMI

3.

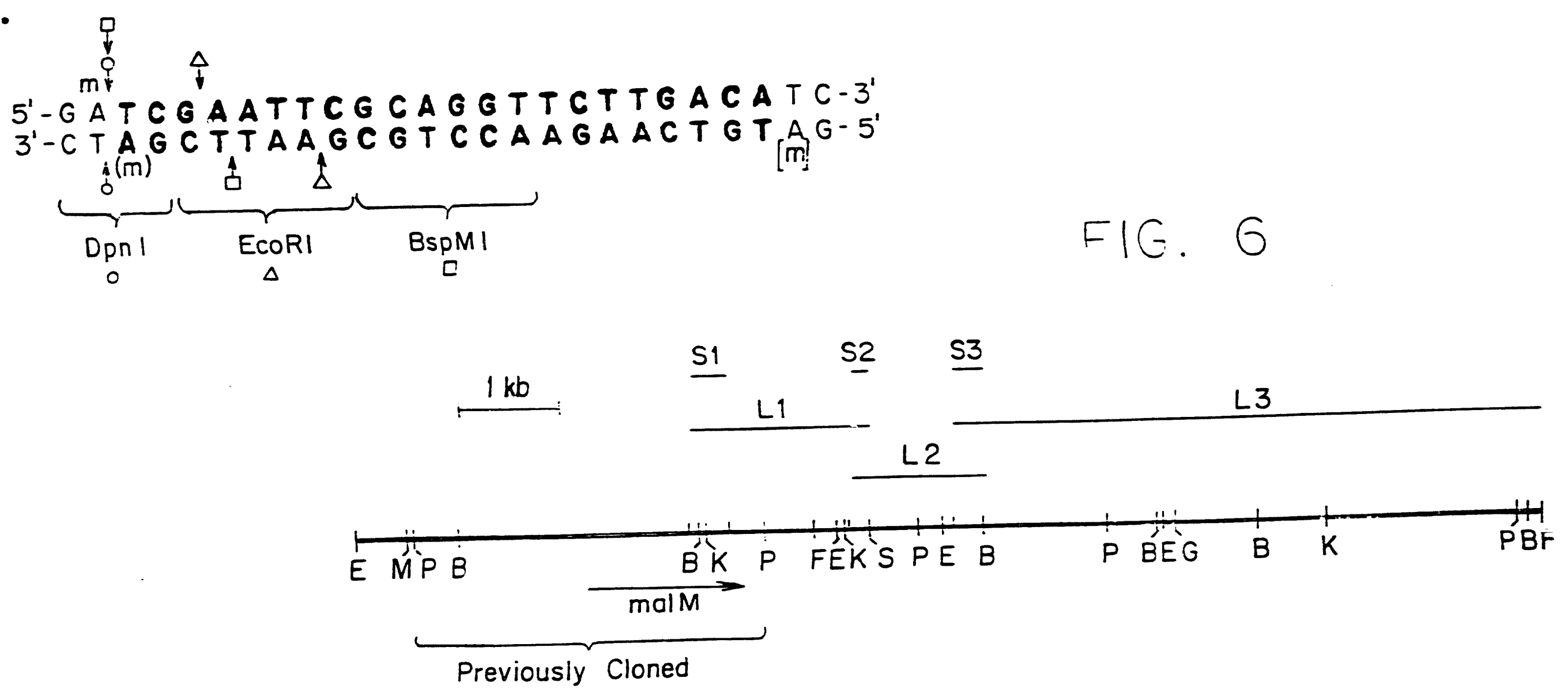



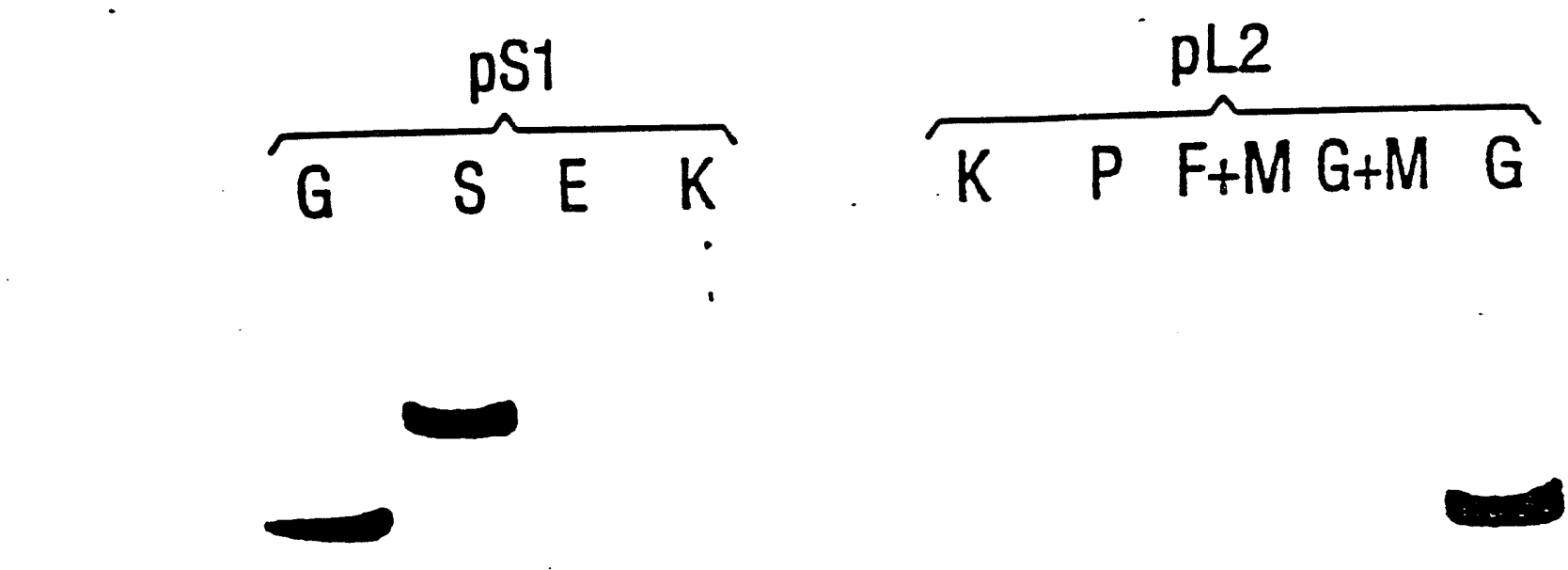

$k b$

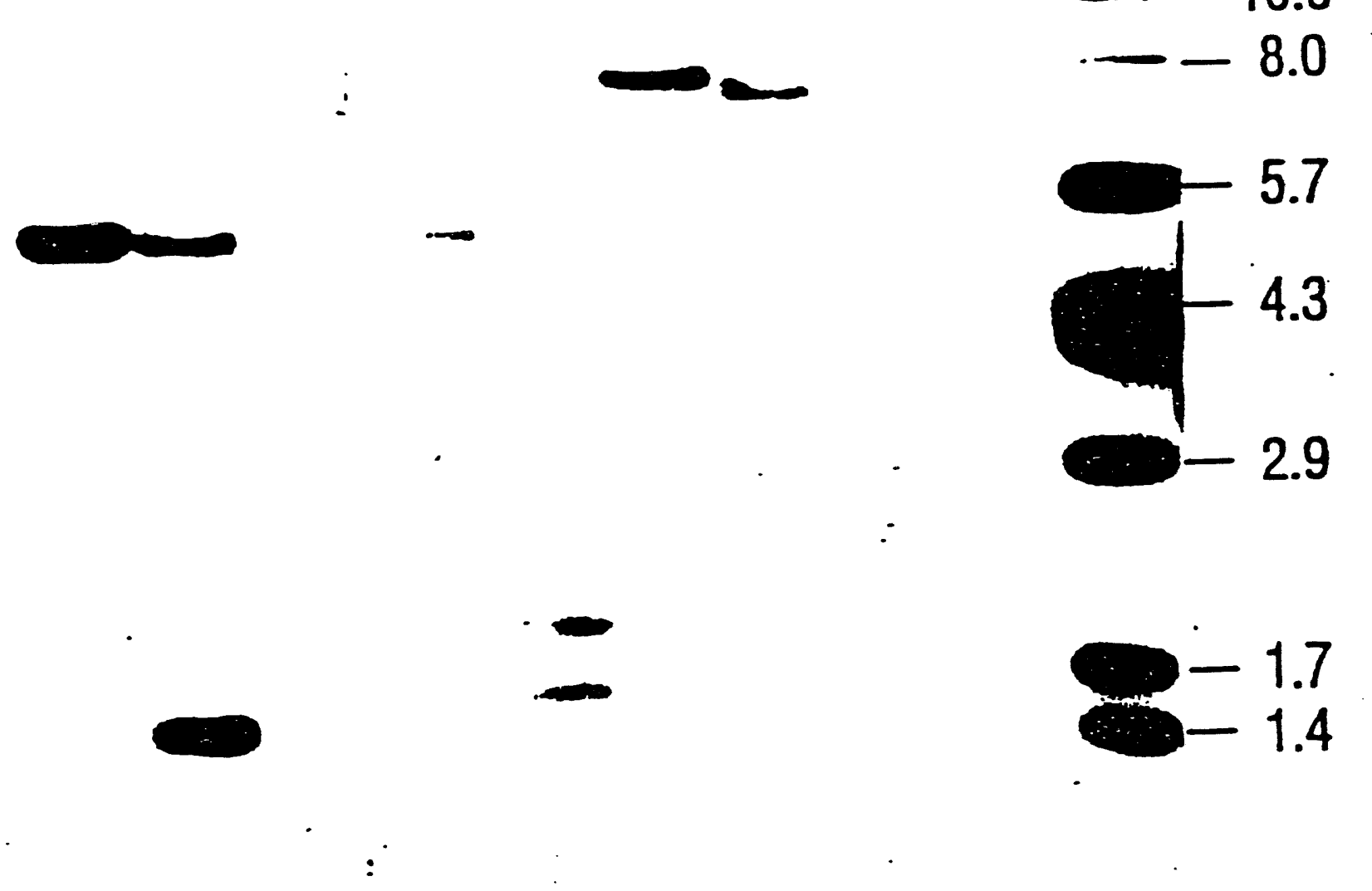

FIG. 7 


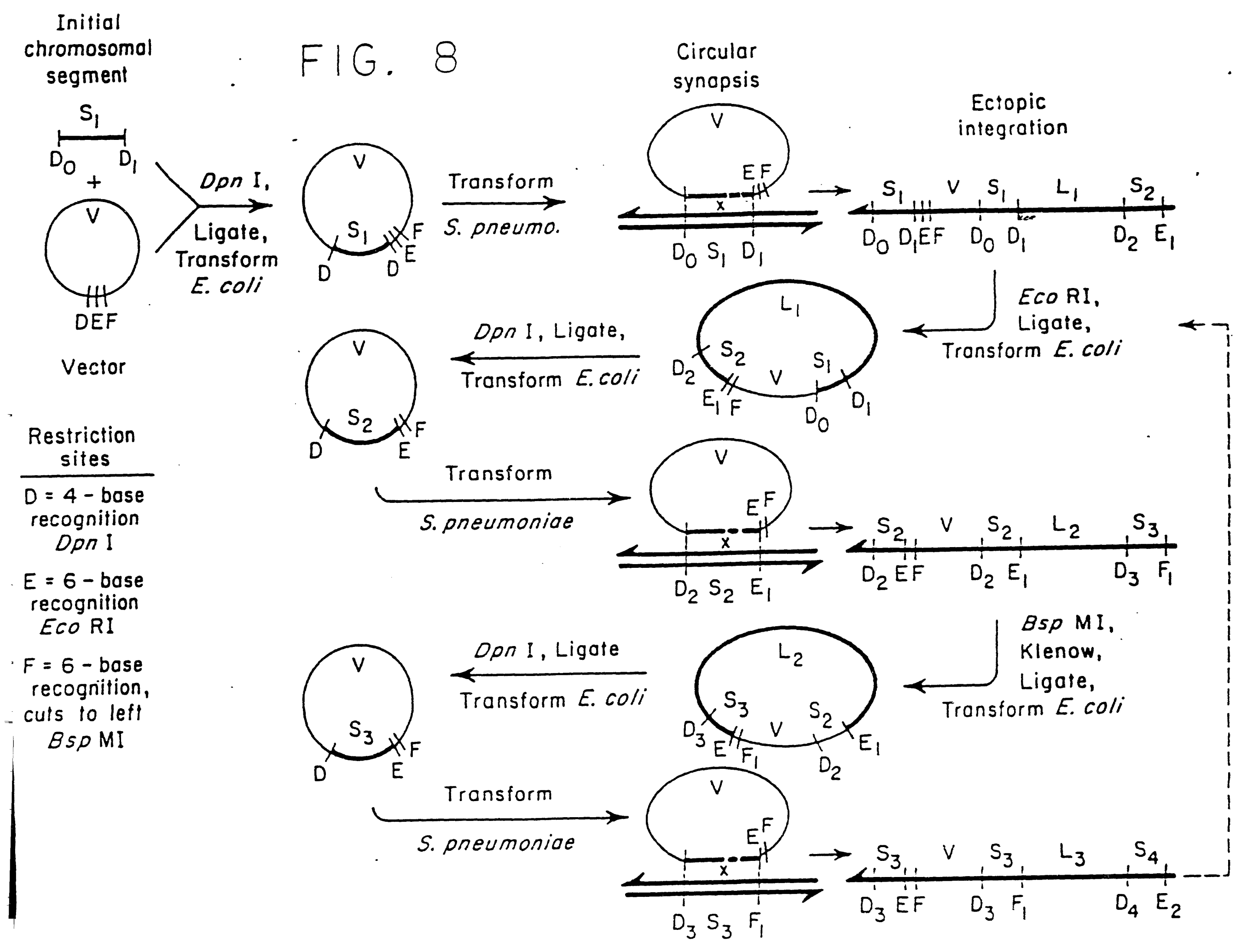


$\ldots$

FIG. 9
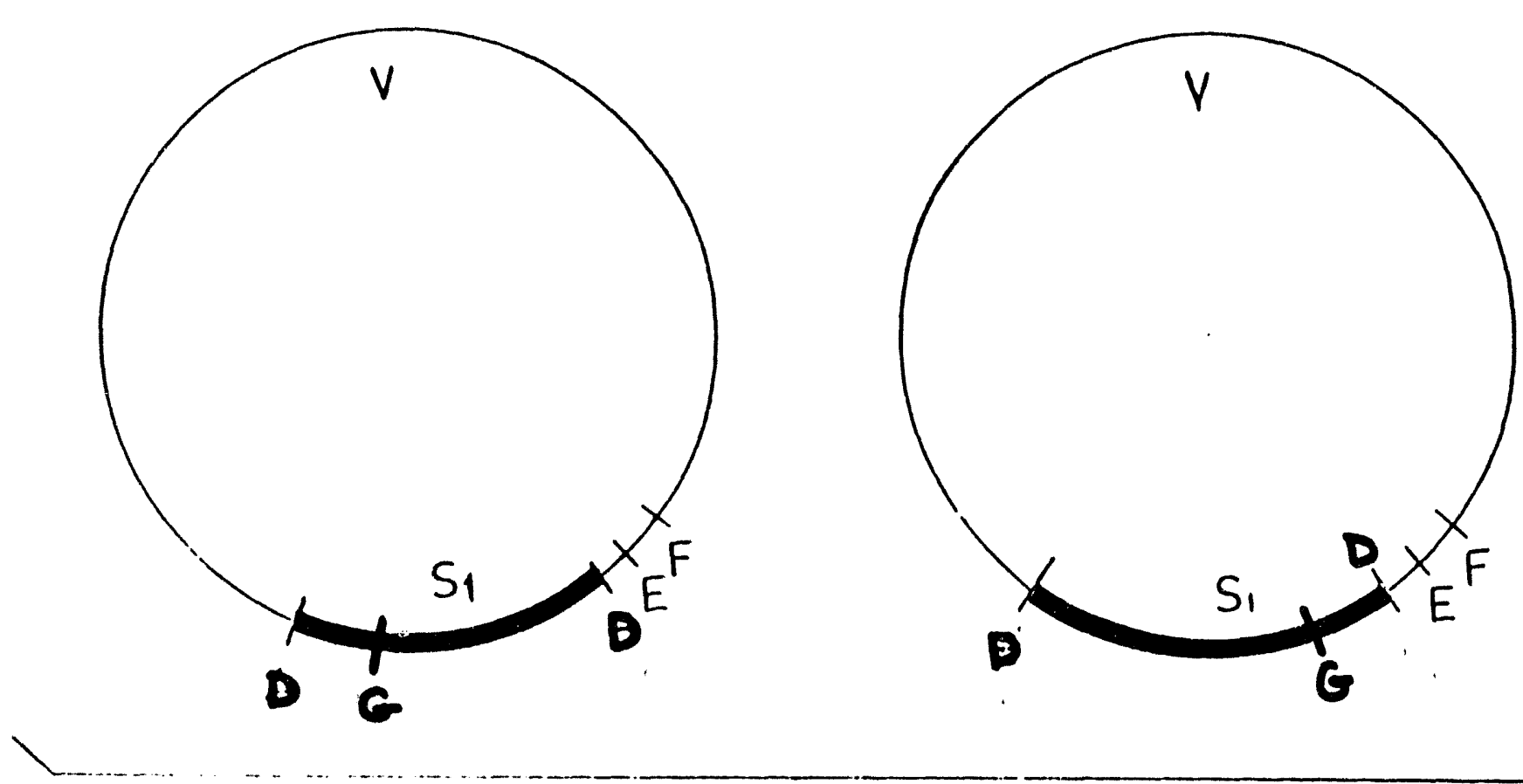

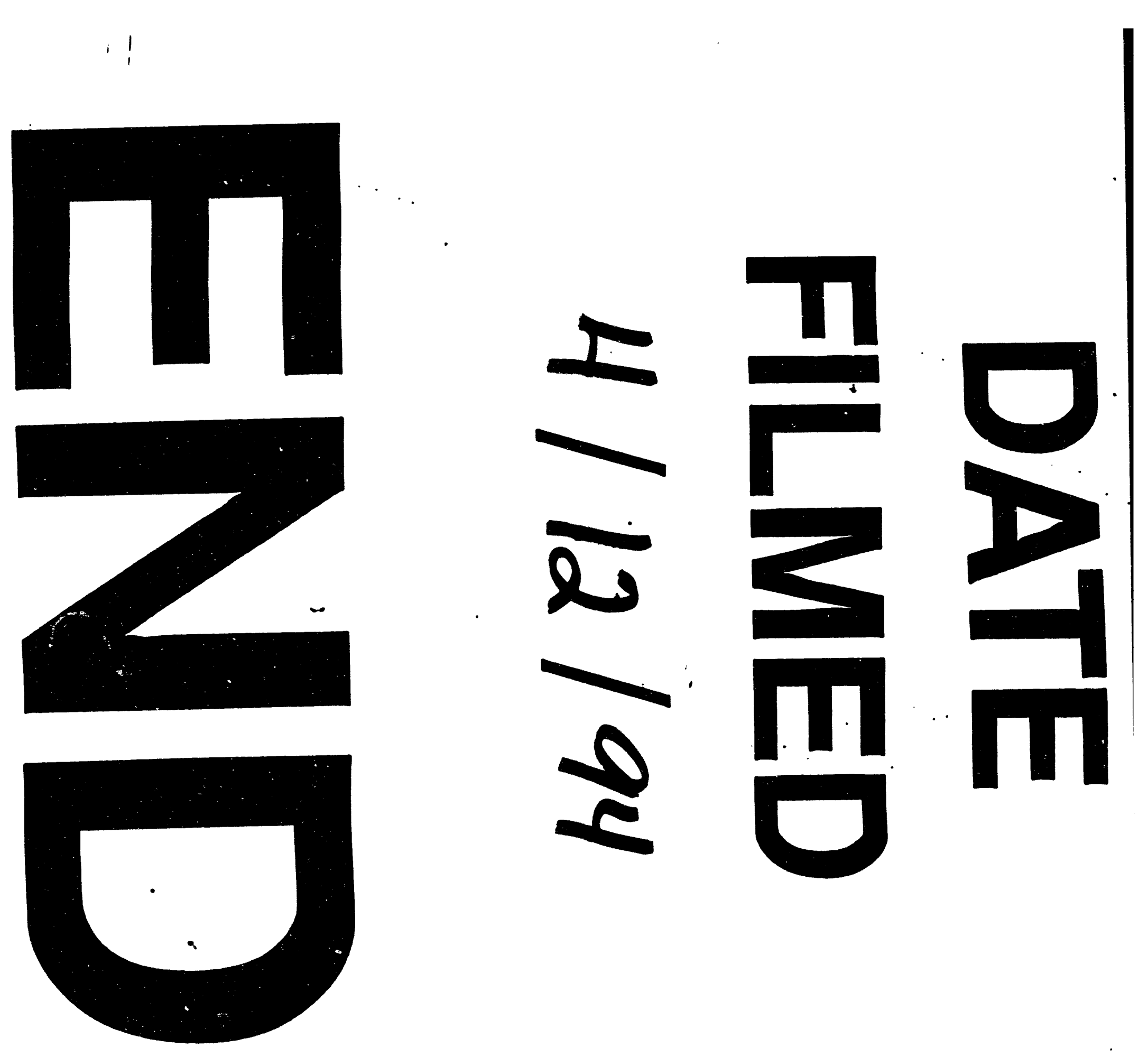
